\title{
Interações potenciais entre medicamentos e medicamentos-álcool em pacientes alcoolistas atendidos por um Centro de Atenção Psicossocial Álcool e Drogas
}

\author{
Potential interactions between drugs and alcohol-medications in alcoholic patients treated by a \\ Psychosocial Alcohol and Drug Care Center \\ Interacciones potenciales entre las drogas y los alcohol-medicaciones en los pacientes alcohólicos \\ tratados por un Centro Psicosocial de Cuidado de Alcohol y las Drogas
}

\section{Resumo}

A dependência química do álcool apresenta fatores limitantes ao tratamento, como a ocorrência de recaídas, falta de adesão terapêutica, transtornos neurológicos associados ou decorrentes do etilismo e efeitos sinérgicos, tóxicos ou nulos do álcool. O presente estudo teve como objetivo identificar as interações potenciais entre medicamentos e medicamento-álcool em pacientes com tratamento de dependência química ao álcool atendidos por um Centro de Atenção Psicossocial Álcool e Drogas. Desta forma foi realizado um estudo observacional do tipo transversal, descritivo, desenvolvido durante experiências vivenciadas no Programa de Educação pelo Trabalho em Saúde, temática Interprofissionalidade. Foram avaliados os dados de 31 pacientes a partir da análise dos prontuários, bem como a avaliação do livro de registros de dispensação de medicamentos psicotrópicos. Verificou-se que os pacientes atendidos na unidade eram em sua maioria do sexo masculino e com idade entre 19 a 59 anos, 48,4\% eram tabagistas e $58,0 \%$ fazem uso de drogas ilícitas. Identificou-se 41 potenciais interações medicamentosas (IM), bem como a ocorrência de interações entre o álcool e os medicamentos prescritos. A avaliação das prescrições, bem como do perfil desses pacientes, demonstrou a importância de se realizar um acompanhamento farmacoterapêutico de pacientes alcoolistas, que estão sujeitos a inúmeras reações resultantes das IM e interações de medicamentos com o álcool, evitando situações de insucesso terapêutico, além de minimizar os eventos adversos que podem ocorrer.

Palavras-chave: Interações medicamentosas; Alcoolismo; Psicotrópicos; Saúde mental.

\section{Abstract}

Chemical dependence on alcohol presents factors limiting treatment such as the occurrence of relapses, lack of therapeutic adherence, neurological disorders associated with or resulting from alcohol and synergistic, toxic or zero 
effects of alcohol. The present study aimed to identify the potential interactions between drugs and drug-alcohol in patients with treatment of chemical dependence on alcohol treated by a Psychosocial Care Center Alcohol and Drugs. Thus, a cross-sectional, descriptive observational study was carried out, developed during experiences experienced in the Health Work Education Program, interprofessionality theme. Data from 31 patients were evaluated from the analysis of medical records, as well as the evaluation of the book of records for dispensing psychotropic drugs. It was found that the patients seen in the unit were mostly male and aged between 19 and 59 years, $48.4 \%$ were smokers and $58.0 \%$ used illicit drugs. We identified 41 potential drug interactions (IM), as well as the occurrence of interactions between alcohol and prescribed medications. The evaluation of prescriptions, as well as the profile of these patients, demonstrated the importance of performing a pharmacotherapeutic follow-up of alcoholic patients, who are subject to numerous reactions resulting from MI and drug interactions with alcohol, avoiding situations of therapeutic failure, besides minimizing adverse events that may occur.

Keywords: Drug interactions; Alcoholism; Psychotropic; Mental health.

\section{Resumen}

La dependencia química del alcohol presenta factores que limitan el tratamiento como la aparición de recaídas, la falta de adherencia terapéutica, los trastornos neurológicos asociados o resultantes del alcohol y los efectos sinérgicos, tóxicos o nulos del alcohol. El presente estudio tuvo como objetivo identificar las posibles interacciones entre las drogas y el alcohol-droga en pacientes con tratamiento de la dependencia química del alcohol tratados por un Centro de Atención Psicosocial alcohol y drogas. Así, se realizó un estudio observacional descriptivo transversal, desarrollado durante las experiencias experimentadas en el Programa de Educación para el Trabajo en Salud, tema interprofesionalidad. Los datos de 31 pacientes fueron evaluados a partir del análisis de las historias clínicas, así como de la evaluación del libro de registros para la dispensación de psicofármacos. Se encontró que los pacientes atendidos en la unidad eran en su mayoría del sexo masculino y tenían entre 19 y 59 años, 48,4\% eran fumadores y 58,0\% utilaban drogas ilícitas. Se identificaron 41 posibles interacciones medicamentosas (IM), así como la aparición de interacciones entre el alcohol y los medicamentos recetados. La evaluación de las prescripciones, así como el perfil de estos pacientes, demostraron la importancia de realizar un seguimiento farmacoterapéutico de los pacientes alcohólicos, que están sujetos a numerosas reacciones resultantes de la IM y las interacciones medicamentosas con el alcohol, evitando situaciones de fracaso terapéutico, además de minimizar los eventos adversos que pueden ocurrir.

Palabras clave: Interacciones farmacológicas; Alcoholismo; Psicotrópico; Salud Mental.

\section{Introdução}

A dependência química do álcool, definida como um transtorno mental, é caracterizada pela perda de controle sobre o consumo, podendo ser observado em indivíduos de diferentes idades e etnias, sendo frequentemente associado ao aumento do risco de acidentes, maiores índices de criminalidade e violência doméstica (Witkiewitz, Litten\&Leggio, 2019; American Psychiatric Association, 2014).

Estudo aponta que até $12 \%$ da população pode ser acometida ao longo da vida pelas consequências do uso do álcool, sendo uma prática bastante difundida, presente desde tempos remotos na sociedade, inserida por meio de inúmeros rituais religiosos e culturais (Zotesso, Paiva \& Marques, 2018). A faixa etária com um maior padrão de consumo de bebidas alcoólicas encontra-se entre 18 e 59 anos, correspondendo a maior parte da população com consumo habitual, ocorrendo um declínio após esta idade, em indivíduos com idade superior a 60 anos (Munhoz et al., 2017).

Os fatores limitantes do tratamento se referem a ocorrência de recaídas pelos usuários ao consumo do álcool, a dificuldade de adesão terapêutica devido às alterações nos efeitos dos medicamentos, os transtornos neurológicos associados ou decorrentes do consumo do álcool e a ação do próprio álcool em induzir efeitos sinérgicos, tóxicos ou nulos (Silva, 2017; Ferreira, Borba, Capistrano, Czarnobay \& Maftum, 2015). O alcoolismo é frequentemente associado a comorbidades psiquiátricas como depressão e transtorno de ansiedade, podendo ser classificado como o fator desencadeante ou um quadro de coexistência, em que um reforça o outro quando não tratados cuidadosamente, o que requer farmacoterapia com o uso de psicotrópicos (Cunha, Iglesias \& Borloti, 2020).

Por ser considerado um problema de saúde pública, medidas foram desenvolvidas para conter o avanço dos índices de alcoolistas, entre elas a criação de espaços de cuidados. Sendo um desses espaços o Centro de Atenção Psicossocial para usuários de Álcool e outras Drogas (CAPS ad), criado de acordo com as premissas da reforma psiquiátrica, o qual conta com um serviço 
de atenção diária (Lacerda \& Fuentes-Rojas, 2016) visando um cuidado integral, singular e coletivo ao paciente com transtornos do uso e dependência de substâncias psicoativas, os quais passam a receber uma terapêutica com abordagem psicossocial (Brasil, 2002). Além disso, o Caps ad é composto por uma equipe multiprofissional com formações acadêmicas diferentes que trabalham juntos, compartilhando práticas, com aprendizado em conjunto para melhorar a qualidade do cuidado em saúde (Anjos et al., 2021).

O álcool pode interagir com diversos medicamentos, incluindo os psicotrópicos como antidepressivos, ansiolíticos e antipsicóticos, que são comumente prescritos para o tratamento de transtornos mentais e do alcoolismo, podendo aumentar o risco de efeitos adversos de um medicamento ao prolongar o efeito e/ou aumentar sua disponibilidade, por outro lado, o fármaco pode aumentar os efeitos tóxicos induzidos pelo álcool (Silva, 2017).

Ademais, os psicotrópicos se encontram ainda dentre os grupos farmacológicos que mais apresentam risco de interação medicamentosa (IM), onde a administração concomitante de dois ou mais medicamentos desta classe pode afetar a absorção, distribuição, metabolismo ou excreção de um deles, podendo reduzir sua biodisponibilidade e promover a potencialização ou anulação do efeito terapêutico esperado (Pasqualotto et al., 2018). Desta forma, o presente estudo teve como objetivo identificar as interações potenciais entre medicamentos e medicamento-álcool por pacientes em tratamento de dependência alcoólica atendidos por um Centro de Atenção Psicossocial Álcool e Drogas.

\section{Metodologia}

\subsection{Tipo de estudo e local da pesquisa}

Estudo observacional, transversal, retrospectivo, descritivo, com uma abordagem quantitativa, realizado no período de setembro de 2020 a março de 2021, desenvolvido durante experiências vivenciadas no Programa de Educação pelo Trabalho para a Saúde - PET Saúde/ Interprofissionalidade.

O local de realização do estudo foi o CAPS ad, unidade que apresenta como área física uma sala de atendimento, farmácia, enfermaria, áreas de oficinas e recepção, e tem como composição da equipe enfermeiros, médico psiquiatra, psicólogo, farmacêutico, oficineiro, agentes administrativos, auxiliar de serviços gerais, profissional de educação física, assistente social, profissional da segurança e coordenação. O território de abrangência consiste na cidade de Jequié, interior da Bahia, possui uma população estimada, em 2020, de 156 mil habitantes de acordo com Instituto Brasileiro de Geografia e Estatística.

\subsection{Amostra e coleta de dados}

Dos 88 pacientes ativos atendidos pelo CAPS ad, foram avaliados os dados de 31 , sendo selecionados todos os pacientes alcoolistas, conforme o objetivo do presente trabalho, que estavam sendo acompanhados pela unidade no período estabelecido para o estudo. Para tanto, foi feita uma avaliação dos prontuários dos usuários atendidos, bem como a avaliação do livro de registro de dispensação de medicamentos psicotrópicos.

\subsection{Instrumento de coleta e variáveis}

Para a coleta foi utilizado um formulário estruturado utilizando critérios pré-estabelecidos no projeto aprovado pelo Comitê de Ética em Pesquisa (Formulário para coleta de dados dos pacientes do CAPS ad). Foram coletadas variáveis sociodemográficas como: idade (19 a 59 anos não idosos $\geq 60$ anos idosos), sexo (feminino e masculino), estado civil (com companheiro e sem companheiro), ocupação (exerce atividade trabalhista e não exerce), escolaridade (analfabeto/ensino fundamental incompleto e ensino fundamental/médio/superior completo), renda familiar mensal (até 1 salário mínimo e 1 a 3 salários mínimos), religião (evangélica/católica e nenhuma) e informações clínicas: tabagista (sim/ não e frequência diária), etilista (sim/ não e frequência diária), uso de drogas ilícitas (sim/ não e quais), farmacoterapia (medicamentos dispensados pelos 
CAPS ad, sob controle especial) e comorbidades dos pacientes em tratamento de alcoolismo que estavam cobertos pela unidade no período do estudo.

Os fármacos em uso pelos pacientes foram classificados pela Anatomical Therapeutic Chemical (ATC) (World Health Organization, 2013) da Organização Mundial da Saúde (OMS), que divide os princípios ativos em diferentes grupos e subgrupos, de acordo com o órgão ou sistema sobre o qual atuam e segundo as suas propriedades químicas, farmacológicas, terapêuticas e substância química, sendo utilizada a classificação do terceiro nível, subgrupo que classifica a substância ativa segundo a sua ação farmacológica (Vidotti, 1993).

A partir dos dados referentes a terapia farmacológica de cada paciente, foi então feita a avaliação das IM potenciais por meio do aplicativo IBM Micromedex Drug Int. ${ }^{\circledR}$, para Android (versão v.2310), que é uma ferramenta de apoio na tomada de decisões, trazendo informações quanto aos medicamentos, toxicologia, doenças, cuidado primário, educação do paciente e medicina alternativa, podendo ser considerada como o padrão de excelência em informação clínica. Segundo essa base de dados, a classificação das interações foi feita de acordo com a gravidade, podendo ser definida como contraindicada, de gravidade maior, moderada, de gravidade menor e desconhecida, ou ainda segundo a documentação descrita no meio científico, sendo então consideradas como excelente, boa, razoável e desconhecida (Healthcare, 2011). Por meio da base também foi verificado o mecanismo de ação envolvendo as IM encontradas, bem como o manejo clínico dos pacientes que foram expostos a elas.

Para a avaliação das possíveis interações álcool/fármaco, foi utilizada a bula do profissional disposto no bulário eletrônico disponível no site da Agência Nacional de Vigilância Sanitária (ANVISA), que apresenta conteúdo sobre educação em saúde, legislação específica sobre o assunto e endereços eletrônicos de interesse na área de saúde, tendo por objetivo facilitar o acesso rápido e gratuito pela população e profissional de saúde às bases de dados das bulas de medicamentos (Agência Nacional de Vigilância Sanitária, sd).

\subsection{Análise dos dados}

O processamento dos dados foi realizado utilizando os programas Microsoft Office Excel ${ }^{\circledR}$ 2013, como banco de dados para transcrever as informações coletadas do livro de registro de dispensação e dos prontuários dos pacientes, e para análise dos dados foi utilizado o programa SPSS Statistics for Windows (IBM SPSS, 21.0, 2012, Armonk, NY: IBM Corp.).

Realizou-se análise descritiva, estimando-se as frequências e porcentagens absolutas e relativas das variáveis categóricas e medindo-se a tendência central (média) e a dispersão (desvio-padrão) das variáveis quantitativas. Para avaliação da distribuição normal das variáveis numéricas foi utilizado o teste de Kolmogorov-Smirnov, considerando $\mathrm{p}<0,05$ distribuição normal.

\subsection{Questões éticas}

A referida pesquisa atendeu a todos os preceitos éticos. O estudo "PET Saúde Interprofissionalidade: ações integradas entre ensino, serviços de saúde e comunidade", da qual este estudo faz parte, foi aprovado pelo CEP da Universidade Estadual do Sudoeste da Bahia, sob número de CAAE 33710120.3.0000.0055.

\section{Resultados}

Foram analisados os prontuários de 31 pacientes em tratamento do alcoolismo. Verificou-se que 90,3\% dos participantes apresentavam idade entre 19 e 59 anos, com média de 46 anos e desvio padrão de $\pm 11,3$. O sexo masculino correspondeu a 77,4\% das pessoas avaliadas, Tabela 1 .

Com relação aos dados clínicos, foi observado que entre os participantes do estudo, 48,4\% eram tabagistas, com frequência de uso do cigarro 3 vezes ou mais na semana. Quanto ao consumo de álcool pelos pacientes estudados, todos faziam uso em maior ou menor frequência semanal, onde $83,9 \%$ consomem bebida alcoólica 3 vezes ou mais na semana. 
Tabela 1 - Características sociodemográficas de pacientes em tratamento para alcoolismo atendidos por um Centro de Atenção Psicossocial Álcool e Drogas - Bahia, Brasil. 2021 (N=31).

\begin{tabular}{|c|c|c|}
\hline Variável & $\mathbf{N}$ & $\%$ \\
\hline \multicolumn{3}{|l|}{ Idade } \\
\hline 19 a 59 anos & 28 & 90,3 \\
\hline$\geq 60$ anos & 3 & 9,7 \\
\hline \multicolumn{3}{|l|}{ Sexo } \\
\hline Masculino & 24 & 77,4 \\
\hline Feminino & 7 & 22,6 \\
\hline \multicolumn{3}{|l|}{ Estado civil } \\
\hline Sem companheiro & 24 & 77,4 \\
\hline Com companheiro & 7 & 22,6 \\
\hline \multicolumn{3}{|l|}{ Ocupação } \\
\hline Exerce atividade trabalhista & 8 & 25,9 \\
\hline Não exerce & 23 & 74,1 \\
\hline \multicolumn{3}{|l|}{ Renda Familiar Mensal } \\
\hline Até 1 salário & 22 & 70,9 \\
\hline 1 a 3 salários & 9 & 29,1 \\
\hline \multicolumn{3}{|l|}{ Reside com } \\
\hline Família/Companheiro & 21 & 67,7 \\
\hline Sozinho & 10 & 32,3 \\
\hline \multicolumn{3}{|l|}{ Religião } \\
\hline Católica/evangélica & 25 & 80,6 \\
\hline Não pratica & 6 & 19,4 \\
\hline \multicolumn{3}{|l|}{ Escolaridade } \\
\hline Analfabeto/EF incompleto & 20 & 64,5 \\
\hline EF completo/médio/ superior & 11 & 35,5 \\
\hline
\end{tabular}

EF: Ensino Fundamental. Fonte: Autores.

No que diz respeito ao uso de drogas ilícitas, 58,0\% dos pacientes fazem uso, sendo a maconha, a cocaína e o crack as drogas citadas pelos usuários, os quais $28,6 \%$ fazem uso das três drogas referidas, enquanto os demais utilizam duas ou menos.

Dentre as pessoas acompanhadas pelo CAPS ad, 32,2\% apontaram alguma comorbidade, onde a hipertensão arterial sistêmica apresentou uma maior frequência, sendo identificada em 50,0\% destes pacientes, seguido de transtornos mentais (depressão, ansiedade e transtorno bipolar) 30,0\% e diabetes mellitus 20,0\%.

Foi identificado o total de 12 medicamentos sendo utilizados pelos pacientes, com média de 3 medicamentos sujeitos a controle especial por paciente e desvio padrão de $\pm 0,95$. Os medicamentos foram codificados pela classificação ATC, utilizando a descrição do nível 3, apresentados na Tabela 2. 
Tabela 2 - Classificação pela Anatomical Therapeutical Chemical e frequência dos medicamentos em uso por pacientes em tratamento para alcoolismo atendidos por um Centro de Atenção Psicossocial Álcool e Drogas - Bahia, Brasil. 2021. (N=95).

\begin{tabular}{cccc}
\hline Medicamento & Fr (\%) & ATC Nível 3 & $\begin{array}{c}\text { Classificação } \\
\text { ATC }\end{array}$ \\
\hline Diazepam & 31,6 & Ansiolítico & N05BA01 \\
Carbamazepina & 14,7 & Antiepilético & N06AA09 \\
Risperidona & 14,7 & Antipsicótico & N05AX08 \\
Fluoxetina & 9,5 & Antidepressivo & N06AB03 \\
Naltrexona & 7,3 & Drogas usadas em transtornos viciantes & N07BB04 \\
Amitriptilina & 6,3 & Antidepressivo & N06AA09 \\
Prometazina & 5,3 & Anti-histamínicos para uso sistêmico & R06AD02 \\
Haloperidol & 3,2 & Antipsicótico & N05AD01 \\
Ácido valpróico & 2,1 & Antiepilético & N03AG01 \\
Topiramato & 2,1 & Antiepilético & N03AX11 \\
Biperideno & 2,1 & Agente anticolinérgico & N04AA02 \\
Paroxetina & 1,1 & Antidepressivo & N06AB05 \\
\hline frequência relativa. ATC: Anatomical Therapeutical Chemical. Fonte: Autores.
\end{tabular}

A partir da avaliação das possíveis interações medicamentosas presentes na farmacoterapia dos pacientes, utilizando o aplicativo do Micromedex ${ }^{\circledR}$, foram encontradas um total de 41 IM potenciais em 23 dos pacientes, sendo 14 diferentes, com média de 1,78 (desvio padrão $\pm 0,95$ ) IM por paciente, Tabela 3. 
Tabela 3 - Perfil e frequência de interações medicamentosas potenciais envolvendo a farmacoterapia de pacientes em tratamento para alcoolismo atendidos por um Centro de Atenção Psicossocial Álcool e Drogas - Bahia, Brasil. 2021. (N=41).

\begin{tabular}{|c|c|c|c|c|}
\hline Medicamentos & $\operatorname{Fr}(\%)$ & Gravidade & Documentação & Resultado da interação \\
\hline Diazepam x Fluoxetina & 22,0 & Menor & Boa & Maiores concentrações séricas de diazepam. \\
\hline Amitriptilina x Diazepam & 14,6 & Moderado & Boa & $\begin{array}{c}\text { Déficits psicomotores, com diminuição do tempo de reação e da } \\
\text { vigilância. }\end{array}$ \\
\hline Carbamazepina $\mathbf{x}$ Risperidona & 12,2 & Moderado & Excelente & Redução da exposição ao risperidone e ao 9-OH-risperione. \\
\hline Fluoxetina x Risperidona & 9,8 & Maior & Boa & $\begin{array}{l}\text { Aumento das concentrações plasmáticas de risperidona e aumento do } \\
\text { risco de prolongamento do intervalo QT. }\end{array}$ \\
\hline Amitriptilina x Carbamazepina & 7,3 & Moderado & Boa & Diminuição da eficácia da amitriptilina. \\
\hline Carbamazepina $x$ Haloperidol & 7,3 & Moderado & Boa & Diminuição das concentrações de haloperidol. \\
\hline Amitriptilina $x$ Risperidona & 4,9 & Maior & Falha & $\begin{array}{l}\text { Risco aumentado de cardiotoxicidade (prolongamento QT, torsades de } \\
\text { pointes, parada cardíaca). }\end{array}$ \\
\hline Carbamazepina x Fluoxetina & 4,9 & Maior & Boa & $\begin{array}{l}\text { Aumento da exposição à carbamazepina e aumento do risco de } \\
\text { toxicidade. }\end{array}$ \\
\hline Haloperidol x Prometazina & 4,9 & Maior & Falha & Risco aumentado de prolongamento do intervalo QT. \\
\hline Amitriptilina x Prometazina & 2,4 & Maior & Falha & Risco aumentado de prolongamento do intervalo QT. \\
\hline Haloperidol x Risperidona & 2,4 & Maior & Falha & Risco aumentado de prolongamento do intervalo QT. \\
\hline Paroxetina $x$ Risperidona & 2,4 & Maior & Boa & $\begin{array}{l}\text { Aumento do risco de prolongamento do intervalo QT e aumento da } \\
\text { exposição à risperidona. }\end{array}$ \\
\hline Amitriptilina $x$ Fluoxetina & 2,4 & Maior & Falha & $\begin{array}{l}\text { Aumento do risco de toxicidade do antidepressivo tricíclico, } \\
\text { prolongamento do intervalo QT e síndrome da serotonina. }\end{array}$ \\
\hline Fluoxetina $x$ Prometazina & 2,4 & Maior & Falha & Risco aumentado de prolongamento do intervalo QT. \\
\hline
\end{tabular}


Foi realizada uma busca na bula do profissional, disponível no Bulário eletrônico da Anvisa, visando identificar os resultados das possíveis interações dos fármacos prescritos e o álcool, sendo obtidos os dados presentes na Tabela 4.

Tabela 4 - Interações potenciais entre álcool e medicamentos utilizados por pacientes em tratamento para alcoolismo atendidos por um Centro de Atenção Psicossocial Álcool e Drogas. Bahia, Brasil. 2021

\begin{tabular}{|c|c|}
\hline Medicamento x Álcool & Resultado da interação \\
\hline Ácido valpróico x Álcool & Pode produzir depressão no sistema nervoso central. \\
\hline Amitriptilina x Álcool & $\begin{array}{l}\text { Potencialização da resposta ao álcool, podendo aumentar o perigo inerente a qualquer } \\
\text { tentativa de suicídio ou superdose. }\end{array}$ \\
\hline Biperideno x Álcool & Aumento do efeito do álcool. \\
\hline Carbamazepina x Álcool & Pode reduzir a tolerância ao álcool. \\
\hline Diazepam x Álcool & $\begin{array}{c}\text { Potencial para aumentar os efeitos clínicos de Diazepam, incluindo possivelmente } \\
\text { sedação grave, que pode resultar em coma ou morte, depressão cardiovascular e/ou } \\
\text { respiratória clinicamente relevantes. }\end{array}$ \\
\hline Fluoxetina x Álcool & $\begin{array}{l}\text { A combinação do álcool com o tratamento com cloridrato de fluoxetina não é } \\
\text { aconselhável. }\end{array}$ \\
\hline Haloperidol x Álcool & Pode reduzir a capacidade de atenção e aumentar a depressão do SNC. \\
\hline Naltrexona x Álcool & $\begin{array}{c}\text { Não foram realizados estudos para avaliar as possíveis interações medicamentosas da } \\
\text { naltrexona com outras substâncias que não os opióides. }\end{array}$ \\
\hline Paroxetina x Álcool & Uso concomitante deste medicamento com álcool não é recomendado. \\
\hline Prometazina x Álcool & Aumento dos efeitos sedativos dos anti-histamínicos H1. \\
\hline Risperidona x Álcool & $\begin{array}{l}\text { Devido a seus efeitos primários sobre o SNC, risperidona deve ser administrada com } \\
\text { cautela em associação com outros medicamentos com ação central ou álcool. }\end{array}$ \\
\hline Topiramato x Álcool & Pode provocar sonolência e tontura. \\
\hline
\end{tabular}

Anvisa: Agência Nacional de Vigilância Sanitária. SNC: Sistema Nervoso Central. Fonte: Autores.

\section{Discussão}

No presente estudo, a partir da avaliação dos dados de 31 pacientes em tratamento para alcoolismo atendidos pelo CAPS ad, foi observada uma prevalência de indivíduos do sexo masculino, com faixa etária variando de 19 a 59 anos, bem como a identificação de potenciais interações medicamentosas e também medicamentos com álcool, que pode ser justificado pelo uso contínuo de medicamentos psicotrópicos e uso recorrente do álcool por recaídas.

O alcoolismo, caracterizado como uma condição complexa e dinâmica que aumenta com episódios de consumo não saudável de álcool, pode apresentar efeitos variados sobre a estrutura e função do cérebro com a idade (Sullivan \& Pfefferbaum, 2019). Esses pacientes, como pôde ser visto neste trabalho, têm muitas dificuldades no tratamento, pois ainda fazem o uso recorrente do álcool mesmo em tratamento para o vício. O CAPS-ad visitado para coleta de dados, dessa forma, utiliza estratégias para diminuir esse consumo durante o tratamento, como a permanência na unidade durante todo dia, diversas atividades que ocupam os pacientes, além da inclusão dos familiares na terapêutica preservando o lugar de fala do usuário. Porém, muitos pacientes estão nesse centro por conta da exigência familiar observada, alimentação, medicação e consultas disponíveis, dificultando o objetivo do tratamento.

A literatura mostra que os jovens e jovens adultos, incluídos na faixa etária que varia dos 18 aos 29 anos, geralmente estão envolvidos em um consumo excessivo e rápido, podendo evoluir para o uso crônico com os passar dos anos, no entanto, tem se observado uma tendência mundial do aumento do consumo entre os adultos mais velhos, bem como um uso abusivo (Damacena et al., 2016; Topiwala, \& Ebmeier, 2018). Em estudo realizado por Malta et al. (2021), foi observado um aumento 
significativo no consumo alcoólico dos indivíduos de 25 a 44 anos e entre aqueles de 55 a 64 anos, o que se assemelha aos achados do presente estudo, onde foi observada uma maior prevalência, entre os pacientes atendimentos na unidade, com média de 49 anos.

No que diz respeito a elevação do número de adultos que fazem uso crônico de álcool, estudos apontam para uma correlação entre os efeitos de problemas econômicos, por exemplo, perda do emprego e a redução do salário, e o aumento do estresse e do sofrimento psicológico causado por estas ocorrências com o aumento da frequência e da quantidade de álcool ingerido (Malta et al., 2021; Zotesso, Paiva \& Marques, 2018), assim como é observado no presente estudo, que apontou maiores porcentagens de pacientes desempregados e com renda mensal menor ou igual a um salário mínimo. Com relação ao índice de idosos alcoolistas, fatores como aposentadoria, perda de amigos, solidão e isolamento social podem torná-los vulneráveis e mais suscetíveis a tal situação (Guidolin et al., 2016).

Ao avaliar o perfil dos pacientes alcoolistas no que diz respeito ao sexo, este estudo indicou um maior padrão de consumo no que se refere a indivíduos do sexo masculino, o mesmo foi observado em pesquisa realizada por Wendt et al. (2021), em que a prevalência total de consumo abusivo de álcool foi maior em indivíduos do sexo masculino e menor no sexo feminino. Esse fato leva a um desarranjo nas relações afetivas, podendo ser vivenciada por meio de violência e sobrecarga à mulher, causada por mudanças comportamentais nos homens alcoólatras (Costa et al., 2020).

Mesmo com um aumento relativo do consumo de álcool pelas mulheres, pesquisas recentes ilustram de forma consistente que os homens bebem mais, e embora os estudos mensurem o consumo excessivo de álcool de várias maneiras e ao longo de vários períodos, a diferença de gênero persiste, sendo essas diferenças atribuídas a fatores biológicos e/ou culturais, podendo ser o resultado de uma combinação desses (Wilsnack, Wilsnack, Gmel \& Kantor, 2018).

No que diz respeito aos efeitos do alcoolismo, a ingestão de bebidas alcoólicas tem sido alvo frequente de discussões devido a aspectos negativos, como o abuso e a dependência, vindo a se tornar uma questão de saúde pública, já que, o uso do álcool de forma abusiva pode acarretar problemas familiares e profissionais, debilidades no organismo, e gerar preconceito, que leva ao isolamento e maior consumo de drogas (Almeida, Fook \& Mariz, 2016; Luna, B. P. L. S., de, Silva, G. L., Jr \& Pereira, I. S. D. S. D., 2019), assim como observado entre os pacientes atendidos na unidade, que também fazem uso de tabaco e de drogas ilícitas, como maconha, crack e cocaína, em maior ou menor frequência.

O consumo crônico do álcool está associado ao aumento do risco de morbimortalidade, devido às complicações cardiovasculares consequentes, bem como o aumento da pressão arterial, no entanto, se faz necessário a avaliação da relação de dependência de outros fatores somados a quantidade ingerida de bebida alcoólica (Almeida, Fook \& Mariz, 2016). Metade dos pacientes que apresentaram comorbidades relataram ter HAS, o que corrobora com os resultados do estudo realizado por Mussi et al. (2018), que aponta que o consumo excessivo de bebidas alcoólicas está associado linearmente com o aumento da pressão arterial.

Outro problema que vem sendo pesquisado se refere a dependência de álcool associado a manifestação de transtornos mentais e de comportamento decorrentes do uso crônico desta substância. A comorbidade de alcoolismo e depressão é prevalente e pode estar associado a alta gravidade, altos custos e mau prognóstico funcional, mais do que qualquer um dos transtornos isoladamente, sendo que, embora as causas e efeitos sejam difíceis de estabelecer, sintomas depressivos geralmente predizem aumento do uso de álcool, e, por outro lado, a recaída no consumo excessivo de álcool prevê uma resposta fraca a tratamento da depressão (Pavkovic et al., 2018).

Mesmo que poucas observações em psiquiatria tenham sido documentadas por tanto tempo e consistentemente quanto a associação entre transtornos mentais e o uso crônico de álcool, foi apontado que até 50\% dos indivíduos recebendo tratamento 
para desintoxicação de álcool também preencheram os critérios diagnósticos para um ou mais transtornos de ansiedade (Anker \& Kushner, 2019), dados que são condizentes aos observados no presente estudo, onde foram identificados pacientes em tratamento para o alcoolismo que haviam sido diagnosticados com depressão, transtorno bipolar ou ansiedade.

$\mathrm{Na}$ literatura, ao se avaliar a correlação existente entre os transtornos depressivos e o alcoolismo foi identificado que ambos compartilham de suscetibilidade genética, mesmo que ainda haja muito a ser compreendido sobre a possível fisiopatologia compartilhada para essas condições, vários sistemas e processos candidatos foram identificados, como disfunção nos sistemas de recompensa e estresse (Connery et al., 2019). Outro ponto se refere a utilização do álcool para lidar com os sintomas dos transtornos mentais, o que aumenta significativamente o risco de dependência alcoólica persistente (Anker \& Kushner, 2019).

Em pacientes que apresentam a associação destas patologias a realização do diagnóstico pode ser desafiador, principalmente devido a sobreposição de sintomas, como os efeitos depressores do álcool, e por causa das características comuns à abstinência do álcool e aos transtornos depressivos, como insônia e agitação psicomotora (Connery et al., 2019). No que diz respeito ao tratamento, a situação pode ser ainda mais complexa, visto que, a ocorrência de depressão em pacientes alcoolistas pode aumentar a gravidade da condição e reduzir a eficácia da farmacoterapia, além disso, o tratamento destas patologias de forma combinada é apontada como uma prática limitada, devido principalmente ao uso reduzido de medicamentos aprovados para o tratamento da dependência, bem como a falta de evidências claras da eficácia dos antidepressivos em pessoas com dependência de álcool (Agabio, Trogu \& Pani, 2018).

Para os pacientes que precisam de medicamentos para tratar a síndrome de abstinência do álcool em ambiente ambulatorial, os benzodiazepínicos são os mais comumente utilizados e representam o padrão ouro, pois são a única classe de medicamentos que não apenas reduz a gravidade da síndrome de abstinência do álcool, mas também reduz o risco de convulsões na abstinência (Witkiewitz, Litten \& Leggio, 2019). Além disso, estudos demonstraram a relevância no tratamento do transtorno do uso do álcool com a naltrexona como tratamento de primeira linha, bem como o topiramato, que vai modular a redução da hiperexcitabilidade do SNC, podendo também ser utilizado como uma das drogas de primeira escolha no tratamento do alcoolismo (Carvalho, Carvalho \& Costa, 2021).

O monitoramento sérico dos medicamentos de pacientes em tratamento para alcoolismo atendidos no CAPS ad se torna essencial diante do número elevado de fármacos prescritos na unidade, bem como as classes mais utilizadas pelos pacientes, os psicofármacos, que vão agir no SNC, podendo apresentar um estreito índice terapêutico, que pode estar associado a IM mais graves (Hiemke et al, 2018), o que requer um acompanhamento farmacoterapêutico rigoroso.

Mesmo que a associação de psicofármacos possa ser benéfica quando proporcionam uma melhor eficácia terapêutica, ela pode se tornar prejudicial quando houver o aparecimento de reações adversas resultante das IM, que correspondem a respostas farmacológicas cujos efeitos se originam pela utilização de um ou mais medicamentos, seja de forma simultânea, ou pela administração anterior de outros medicamentos, ou diminuição do efeito de um ou ambos os fármacos, podendo aumentar o risco de ineficiência do tratamento, ocasionando consequências graves ao paciente (Bosetto, Silva \& Peder, 2020; Silva, Rodrigues, Silva \& Morais, 2019).

As IM que envolvem psicotrópicos comprometem principalmente o processo de metabolização dos fármacos, podendo aumentar consideravelmente o risco de intoxicação, devido à inibição/indução de enzimas metabolizadoras de drogas, por exemplo, as do citocromo P 450. Tais alterações metabólicas podem exacerbar reações adversas, como as que ocorrem no uso de vários antipsicóticos, quando há aumento dos níveis séricos do princípio ativo ou ainda desencadear uma redução na concentração plasmática comprometendo a resposta terapêutica de uma série de drogas, alterando assim os resultados que o paciente necessita (Silva, Lima \& Ruas, 2018). 
Research, Society andDevelopment, v. 10, n. 9, e20610917697, 2021

(CC BY 4.0) | ISSN 2525-3409 | DOI: http://dx.doi.org/10.33448/rsd-v10i9.17697

Dos medicamentos analisados, foram encontradas 14 diferentes associações farmacológicas consideradas potenciais a ocorrência de IM, onde nove apresentaram gravidade maior considerando-se as evidências científicas boas ou falhas, o que aponta para a necessidade de identificar tais interações, principalmente no que se refere a segurança do paciente.

A interação medicamentosa mais frequente foi entre a fluoxetina, um antidepressivo inibidor seletivo da recaptação de serotonina (ISRS), que representa uma classe quimicamente diversa de agentes, cuja principal ação consiste no bloqueio da recaptação desse neurotransmissor, aumentando e prolongando a ação serotoninérgica, associado ao diazepam, um benzodiazepínico que atua nos receptores GABAA (Katzung \& Trevor, 2017; Brunton, Hilal \& Knollmann, 2018). A interação pelo uso concomitante destes fármacos está relacionada a capacidade da fluoxetina em inibir a isoenzima do citocromo P450, responsável pelo metabolismo de diazepam e seu principal metabólito (princípio ativo), resultando em maiores concentrações séricas de diazepam (Diazepam, sd; Diazepam \& Fluoxetine Hydrochloride, 2018). Quando a fluoxetina e o diazepam são administrados concomitantemente, é importante que se monitore os pacientes quanto a sinais e sintomas de concentrações excessivas de diazepam, que pode resultar em sedação, tontura, diminuição da cognição ou desempenho motor (Diazepam \& Fluoxetine Hydrochloride, 2018).

A amitriptilina, um antidepressivo tricíclico cuja ação farmacológica consiste no antagonismo dos transportadores de serotonina (SERT) e de norepinefrina (NET), além de serem antagonistas potentes dos receptores de histamina H1, o que contribui para os seus efeitos sedativos (Katzung \& Trevor, 2017; Brunton, Hilal \& Knollmann, 2018), quando associada ao diazepam, também apresentou frequência relevante de ocorrência. O uso concomitante destes fármacos, ambos agentes depressores do SNC, pode resultar em efeitos aditivos, por meio da ocorrência de déficits psicomotores, com diminuição da vigilância e do tempo de reação (Katzung \& Trevor, 2017; Amitriptyline Hydrochloride \& Diazepam, 2018). Caso a associação destes fármacos seja necessária, é fundamental que o paciente esteja ciente de que pode apresentar comprometimento psicomotor aditivo, o que pode afetar a direção ou outras tarefas que requerem habilidades motoras complexas (Amitriptyline Hydrochloride \& Diazepam, 2018).

Outra interação que apresentou uma frequência considerável foi a associação entre a risperidona, fármaco antipsicóticos atípico, antagonista monoaminérgico seletivo com uma alta afinidade pelos receptores serotoninérgicos 5-HT2 e dopaminérgicos D2, e a carbamazepina, anticonvulsivante que age bloqueando os canais de sódio $\left(\mathrm{Na}^{+}\right)$em concentrações terapêuticas, atuando contra convulsões por eletrochoque máximo (Katzung \& Trevor, 2017; Brunton, Hilal \& Knollmann, 2018).

As IM que envolvem a carbamazepina estão relacionadas com as propriedades do fármaco de indução das enzimas hepáticas, desta forma, o aumento da capacidade metabólica dessas enzimas pela carbamazepina pode causar um aumento na taxa de metabolismo da risperidona mediado pelo citocromo P450, resultando na redução da biodisponibilidade deste fármaco (Katzung \& Trevor, 2017; Risperidone \& Carbamazepine, 2018). Pacientes em uso destes medicamentos devem ser constantemente avaliados, e sempre que necessário, deve ser feito o ajuste da terapia, com aumento da dose da risperidona, seguido da diminuição da carbamazepina até que seja descontinuada, devendo então ajustar a dose da risperidona com diminuição conforme necessário (Risperidone \& Carbamazepine, 2018).

A associação entre a fluoxetina e a risperidona foi a interação de gravidade maior que ocorreu com maior frequência. A maioria dos antipsicóticos estão relacionados com os efeitos de prolongamento QT, medida eletrocardiográfica de despolarização (onda Q) e repolarização ventricular (onda T), tanto em dose terapêutica como em sobredosagem (Rodríguez, Tur, \& Quiles, 2018; Bosetto, Silva \& Peder, 2020). Devido a ação da fluoxetina em inibir o metabolismo da risperidona, com aumento das concentrações plasmáticas dessa, o uso concomitante destes fármacos poderá acarretar efeitos aditivos de prolongamento do intervalo QT, que pode criar um ambiente eletrofisiológico que favorece o aparecimento de arritmias cardíacas que podem 
Research, Society andDevelopment, v. 10, n. 9, e20610917697, 2021

(CC BY 4.0) | ISSN 2525-3409 | DOI: http://dx.doi.org/10.33448/rsd-v10i9.17697

resultar em fibrilação ventricular levando a morte súbita (Fluoxetine, Hydrochloride \& Risperidone, 2018; Rodríguez, Tur, \& Quiles, 2018; Bosetto, Silva \& Peder, 2020). Caso o uso concomitante destes fármacos seja necessário, deve ser feito o acompanhamento farmacoterapêutico, com realização periódica do eletrocardiograma (ECG) e redução da dosagem de risperidona conforme resposta do paciente (Fluoxetine, Hydrochloride \& Risperidone, 2018).

Ademais, as IM devem ser avaliadas e acompanhadas, visto que, a ocorrência clínica dessas nem sempre podem ser controladas somente com a manutenção da prescrição, como o ajuste de doses, suspensão da combinação de fármacos e o monitoramento de possíveis efeitos adversos, fazendo-se necessário uma avaliação individual de riscos e benefícios em conjunto com o paciente e a equipe multiprofissional de referência do projeto terapêutico singular.

O álcool pode interagir com diversos medicamentos de venda livre e controlados, incluindo medicamentos psicotrópicos, sendo que, a interação pode ser de natureza farmacocinética, causando alteração da biodisponibilidade do fármaco, ou farmacodinâmica, que resulta no aumento ou redução da resposta biológica dos fármacos (Oliveira, 2018).

O álcool é metabolizado pela enzima álcool desidrogenase (ADH) e a isoenzima CYP2E1 do citocromo P450, responsável pela metabolização e biotransformação de inúmeros fármacos. O consumo agudo de álcool pode levar a inibição de enzimas envolvidas no seu próprio metabolismo e consequente diminuição do metabolismo dos fármacos, resultando em aumento de sua concentração com sobredosagem, de outro modo, o uso crônico de álcool pode levar a indução enzimática (isoformas de citocromo p450) e consequente aumento do metabolismo, resultando em níveis plasmáticos mais baixos do fármaco na forma in natura (Chan \& Anderson, 2014; Johnson \& Seneviratne, 2014; Guerzoni, Pellesi, Pini \& Caputo, 2018). Além disso, a interação também pode estar relacionada com o mecanismo inverso, onde o medicamento altera o metabolismo do álcool, aumentando os efeitos adversos relacionados ao álcool e diminuindo o limiar de intoxicação (Cheng, Mithoowani, Ungar \& Lee, 2018).

Outra interação entre fármacos psicotrópicos e o álcool está relacionada a ação do álcool como depressor primário do SNC, que pode ocorrer em qualquer nível do neuroeixo, incluído encéfalo, nervos e músculos, sendo que, o mecanismo de inibição das funções neurofisiológicas ligada ao consumo de álcool está relacionado com os efeitos depressivos e sedativos nos receptores GABA-A e no sistema nervoso central (Lemos, Andrade, \& Almeida, 2017).

A associação do álcool a fármacos que também apresentam efeito depressor do SNC, como os benzodiazepínicos, antihistamínicos, anticonvulsivantes e antidepressivos, pode resultar em depressão excessiva do SNC e função psicomotora prejudicada, potencializando a ação sedativa destes, o que pode comprometer a condução de veículos, conduzir a desequilíbrios, perigo de quedas ou até mesmo à morte (Guerzoni, Pellesi, Pini\& Caputo, 2018). Muitos dos pacientes atendidos na unidade em que o presente estudo foi realizado fazem uso de um ou mais medicamentos dos quais foram encontradas evidências da ocorrência de interação quando combinado com o álcool.

Os dados apresentados acima devem ser analisados considerando algumas limitações, já que, o número reduzido de pacientes considerados no estudo pode não ser uma amostra representativa do que é observado na população-alvo, podendo ser encontrado resultados que se referem apenas a população em questão, ou ainda, o perfil desses pode não refletir a situação de pacientes atendidos por outros CAPS ad. Vale apontar também que as possíveis interações identificadas no estudo não foram investigadas clinicamente a fim de verificar a ocorrência de danos ao paciente. Como recomendação para estudos futuros com essa temática, deverá ser feita a verificação da relevância clínica dessas possíveis interações, bem como o acompanhamento farmacoterapêutico dos pacientes polimedicados com psicofármacos, além disso, é importante que se aumente a amostra utilizada, bem como a aplicação de outros métodos que possibilitem o recolhimento de dados pretendidos. 
Research, Society andDevelopment, v. 10, n. 9, e20610917697, 2021

(CC BY 4.0) | ISSN 2525-3409 | DOI: http://dx.doi.org/10.33448/rsd-v10i9.17697

O álcool contribui fortemente na instalação e manutenção de vários problemas sociais, familiares, econômicos e de saúde enfrentados em todo país, com isso esse estudo apresenta uma alta relevância para área da saúde por se tratar de um tema antigo, porém recorrente, e mostra a necessidade de se dar mais ênfase aos estudos sobre essa questão além de mostrar dados epidemiológicos no Brasil e no mundo, não só com a ampliação, como também a renovação sistemática dessas pesquisas. Além disso, os pacientes em uso concomitante de psicotrópicos e álcool estão vulneráveis a acidentes devido efeitos resultantes da interação farmacológica, o que demonstra a necessidade de se avaliar os riscos e o acompanhamento farmacoterapêutico como parte das ações e intervenções psicossociais multiprofissionais da equipe da unidade, assim como da rede intersetorial nos cuidados necessários ao atendimento de pessoas em uso abusivo de álcool.

\section{Conclusão}

Baseado nos dados citados anteriormente, identificou-se potenciais interações medicamentosas envolvendo os psicotrópicos, sendo observado um grande número de IM consideradas de gravidade maior. A avaliação das prescrições demonstrou a importância de se realizar um acompanhamento farmacoterapêutico desses pacientes, que estão sujeitos a inúmeras reações resultantes das IM, evitando situações de insucesso terapêutico, além de minimizar os efeitos que poderiam ocorrer. Além disso, o conhecimento quanto às possíveis reações decorrentes da associação entre o álcool e os fármacos prescritos poderá auxiliar na realização de intervenções visando a diminuição dos riscos dos quais estes pacientes estão expostos, garantindo um tratamento efetivo e seguro.

\section{Referências}

Agabio, R., Trogu, E., \&Pani, P. P. (2018). Antidepressants for thetreatmentofpeoplewithco-occurringdepressionandalcoholdependence. Cochrane data base of systematic reviews, (4).

Agência Nacional de Vigilância Sanitária. Bulário Eletrônico. <https://consultas.anvisa.gov.br/\#/bulario/>

Almeida, T. S. O., de, Fook, S. M. L., \& Mariz, S. R. (2016). Associação entre etilismo e subsequente hipertensão arterial sistêmica: uma revisão sistematizada. Revista Saúde \& Ciência Online, 5(1), 76-90.

American Psychiatric Association. (2014). DSM-5: Manual diagnóstico e estatístico de transtornos mentais. Artmed Editora.

Amitriptyline Hydrochloride \& Diazepam. (2018). In Micromedex: Drug Interactions para Android (versão v2310) [software de aplicativo móvel]. Greenwood Village (CO): IBM Corporation; <http://play.google.com>. É necessária uma assinatura para ver.

Anker, J. J., \& Kushner, M. G. (2019). Co-occurring alcohol use disorder and anxiety: bridging psychiatric, psychological, and neurobiological perspectives. Alcoholresearch: current reviews, $40(1)$.

Anjos A. C.B., Barbosa A.A., Silva A. O., Cunha A. P. S., Silva C. H., Rolim I. A. A., Oliveira P. S., Barros V. S., Santos S. S., Lemos G. S. (2021) A interprofissionalidade e suas percepções: um estudo com participantes do programa de educação pelo trabalho para a saúde

Brasil. Ministério da Saúde. Portaria n 336 de 19 de fevereiro de 2002.<https://bvsms.saude.gov.br/bvs/saudelegis/gm/2002/prt0336_19_02_2002.html>

Bosetto, A., Silva, C. M. da, \& Peder, L. D. (2020). Interações medicamentosas entre psicofármacos e a relação com perfil de prescritores e usuários. Journal health npeps, 5(1), 186-206.

Brunton, L. L., Hilal, -D. R., \& Knollmann, B. C. (2018). As Bases Farmacológicas da Terapêutica de Goodman e Gilman-13. Artmed Editora.

Carvalho, C. S. L., Carvalho, G. S., \& Costa, N. C. (2021). Avanços no tratamento farmacológico do alcoolismo: revisão integrativa. Brazilian Journal of Development, 7(1), 11271-11283.

Chan, L. N. \& Anderson, G. D. (2014). Interações farmacocinéticas e farmacodinâmicas de medicamentos com etanol (álcool). Clinical pharmacokinetics, 53 (12), 1115-1136.

Cheng, C., Mithoowani, F., Ungar, T., \& Lee, M. (2018). Interaction between psychotropic medications and alcohol: Perceptions among patients attending an adult mental health day hospital program. The Canadian journal of hospital pharmacy, 71(1), 7. 
Research, Society andDevelopment, v. 10, n. 9, e20610917697, 2021

(CC BY 4.0) | ISSN 2525-3409 | DOI: http://dx.doi.org/10.33448/rsd-v10i9.17697

Connery, H. S., Taghian, N., Kim, J., Griffin, M., Rockett, I. R., Weiss, R. D., \& McHugh, R. K. (2019). Motivações suicidas relatadas por sobreviventes de overdose de opioides: Um estudo transversal de adultos com transtorno do uso de opioides. Dependência de drogas e álcool, $205,107612$.

Costa,L. C., Meira,E. C., Souza, A. S., Vieira,L. O., Botelho, P. B. \& Rodrigues, V. P. (2020). Memory of women living with alcoholian ellows. Research, Society and Development 9(7): 1-17, e551974268.

Cunha, B. J. G. da, Iglesias, A., \& Borloti, E. B. (2020). Comorbidade entre uso de álcool e outras drogas, transtornos psiquiátricos e comportamento suicida: uma revisão. Revista Psicologia e Saúde.

Damacena, G. N., Malta, D. C., Boccolini, C. S., Souza Júnior, P. R. B. D., Almeida, W. D. S. D., Ribeiro, L. S., \& Szwarcwald, C. L. (2016). Consumo abusivo de álcool e envolvimento em acidentes de trânsito na população brasileira, 2013. Ciência \& Saúde Coletiva, 21, 3777-3786.

Diazepam \& Fluoxetine Hydrochloride. (2018). In Micromedex: Drug Interactions para Android (versão v2310) [software de aplicativo móvel]. Greenwood Village (CO): IBM Corporation; Disponível em <http://play.google.com>. É necessária uma assinatura para ver

Diazepam [Bula]. Minas Gerais: Pharlab Indústria Farmacêutica S.A

Ferreira, A. C. Z., Borba, L. D. O., Capistrano, F. C., Czarnobay, J., \& Maftum, M. A. (2015). Fatores que interferem na adesão ao tratamento de dependência química: percepção de profissionais de saúde. Revista Mineira de Enfermagem, 19(2), 150-164.

Fluoxetine Hydrochloride \& Risperidone. (2018). In Micromedex: Drug Interactions para Android (versão v2310) [software de aplicativo móvel]. Greenwood Village (CO): IBM Corporation; Disponível em <http://play.google.com〉. É necessária uma assinatura para ver.

Guerzoni, S., Pellesi, L., Pini, L. A., \& Caputo, F. (2018). Drug-drug interactions in the treatment for alcohol use disorders: a comprehensive review. Pharmacological research, 133, 65-76.

Guidolin, B. L, Silva, I. G. D., Filho. Nogueira, E. L., Ribeiro, F. P., Jr \& Cataldo, A., Neto. (2016). Padrões de uso de álcool em uma amostra de idosos cadastrados no Programa de Estratégia Saúde da Família de Porto Alegre, Brasil. Ciência \& coletiva saúde, 21, 27-35.

Healthcare, Thomson. (2011). "Micromedex Healthcare Series." Greenwood Village, Colo., Thomson Reuters Healthcare. 11: 1-22.

Hiemke, C., Bergemann, N., Clement, H. W., Conca, A., Deckert, J., Domschke, K., \& Baumann, P. (2018). Consensus guidelines for therapeutic drug monitoring in neuropsychopharmacology: update 2017. Pharmaco psychiatry, 51(01/02), 9-62

Instituto Brasileiro de Geografia e Estatística. Jequié. Disponível em: <https://cidades.ibge.gov.br/brasil/ba/jequie/panorama> Visto em 14/07/21 às 12:26.

Johnson, B. A., \& Seneviratne, C. (2014). Alcohol-medical drug interactions. Handbook of clinical neurology, 125, 543-559.

Katzung, B. G., \& Trevor, A. J. (2017). Farmacologia Básica e Clínica-13. McGraw Hill Brasil.

Lacerda, C. D. B., \& Fuentes-Rojas, M. (2016). Significados e sentidos atribuídos ao Centro de Atenção Psicossocial Álcool e outras Drogas (CAPS AD) por seus usuários: um estudo de caso. Interface-Comunicação, Saúde, Educação, 21, 363-372.

Lemos, A. J. G., Andrade, S. P., \& Almeida, M. M. S., de. (2017). Efeitos toxicológicos desencadeados pelo etanol no organismo humano: uma revisão. Scire Salutis, 7(2), 1-9.

Luna, B. P. L. S, de, Silva, G. L., Jr \& Pereira, I. S. D. S. D. (2019). Alcoholism and comorbidities in women. Journal health npeps, 4(1), 62-79.

Malta, D. C., Silva, A. G. D., Prates, E. J. S., Alves, F. T. A., Cristo, E. B. \& Machado, Í. E. (2021). Convergência no consumo abusivo de álcool nas capitais brasileiras entre sexos, 2006 a 2019: o que dizem os inquéritos populacionais. Revista Brasileira de Epidemiologia, 24.

Munhoz, T. N., Santos, I. S., Nunes, B. P., Mola, C. L. D., Silva, I. C. M. D., \& Matijasevich, A. (2017). Tendências de consumo abusivo de álcool nas capitais brasileiras entre os anos de 2006 a 2013: análise das informações do VIGITEL. Cadernos de Saúde Pública, 33, e00104516.

Mussi, F. C., Portela, P. P., Barretto, L. E. S., Gama, G. G. G., Mendes, A. S., \& Macêdo, T. T. S., de (2018). Consumo de bebida alcoólica e tabagismo em homens hipertensos. Revista Baiana de Enfermagem32, .

Oliveira, A. C. D., Neto. (2018). Interação álcool x medicamento: uma revisão da literatura.

Pasqualotto, A., Bitencourt, P. E. R., Nietiedt, N. A., Paula, L. F. D., Nogueira, R. D. O., \& Gomez, R. (2018). Interações entre medicamentos sujeitos a controle especial dispensados na Farmácia Distrital Centro de Porto Alegre, RS, Brasil. Infarma. Brasília. Vol. 30, n. 3 (2018), p. 146-151.

Pavkovic, B., Zaric, M., Markovic, M., Klacar, M., Huljic, A., \& Caricic, A. (2018). Double screening for dual disorder, alcoholism and depression. Psychiatry research, 270, 483-489.

Risperidone \& Carbamazepine. (2018). In Micromedex: Drug Interactions para Android (versão v2310) [software de aplicativo móvel]. Green wood Village (CO): IBM Corporation; 〈http://play.google.com>.

Rodríguez, -M. M., Tur, F. R., \& Quiles, J. (2018). Medicamentos que afectan al intervalo QT. FMC-Formación Médica Continuada em Atención Primaria, 25(5), 302-307. 
Research, Society andDevelopment, v. 10, n. 9, e20610917697, 2021

(CC BY 4.0) | ISSN 2525-3409 | DOI: http://dx.doi.org/10.33448/rsd-v10i9.17697

Silva, A. J. H. da, Rodrigues, A. C. R., Silva, J. L. da \& Morais, I. C. O. de. (2019). Interações medicamentosas entre psicofármacos em um centro de atenção psicossocial. Encontro de Extensão, Docência e Iniciação Científica (EEDIC), 6.

Silva, S. N., Lima, M. G., \& Ruas, C. M. (2018). Analysis of Potential Drug Interactions in Brazilian Mental Health Services: Prevalence and Associated Factors. Journalof Young Pharmacists, 10(2), 237.

Silva, S. V. L. (2017). A interação do álcool com medicamentos e seus efeitos no organismo. Monografia de Graduação em Farmácia. Faculdade de Educação e Meio Ambiente.

Sullivan, E. V., \& Pfefferbaum, A. (2019). Brain-behavior relations and effects of aging and common comorbidities in alcohol use disorder: A review. Neuropsychology, 33(6), 760 .

Topiwala, A., \& Ebmeier, K. P. (2018). Effects of drink in gon late-life brain and cognition. Evidence-based mental health, $21(1), 12-15$.

Vidotti, C. C. F. (1993). Sistema de Classificação Anatômico Terapêutico Químico (ATC). Infarma-Ciências Farmacêuticas, 2(6), $12-15$.

Wendt, A., Costa, C. S., Costa, F. S., Malta, D. C., \& Crochemore-Silva, I. (2021). Análise temporal da desigualdade em escolaridade no tabagismo e consumo abusivo de álcool nas capitais brasileiras. Cadernos de Saúde Pública, 37, e00050120.

Wilsnack, R. W., Wilsnack, S. C., Gmel, G., \& Kantor, L. W. (2018). Gender differences in binge drinking: Prevalence, predictors, and consequences. Alcohol research: current reviews.

Witkiewitz, K., Litten, R. Z., \&Leggio, L. (2019). Advances in the science and treatment of alcohol use disorder. Science advances, 5(9), eaax4043.

World Health Organization. (2013). Collaborating centre for drug statistics methodology. Guidelines for ATC classification and DDD assignment, 3.

Zotesso, M. C., Paiva, S. M. A., \& Marques, L. O. (2018). Consumo, dependência e caracterização de usuários de álcool em um centro de atenção psicossocial de álcool e drogas. Revista Interdisciplinar de Estudos em Saúde, 430-439. 